\title{
A Trend Model of Internet Public Opinion
}

\author{
Qing Lu \\ Linyi University, Shandong, China \\ luqing@lyu.edu.cn
}

Keywords: public opinion, Internet public opinion, trend model.

Abstract. In modern society, internet information presents rapidly development, and mass media is playing an increasingly important role in spreading public opinion. The interpersonal relationship network is the carrier of public opinion of interpersonal communication, the scale-free network model constructs the interpersonal relationship network topology using BA, through the local interaction rules design of the individual, the individual factors and the external impact of the media construction of free communication between two opposing opinion. Then construct the mutual influence of public opinion and network structure of adaptive public opinion should be the evolution of competition model, propagation member two opposing opinion. Propagate through opposite opinion free communication and confrontation can be better simulated opposite opinion.

\section{Introduction}

Public opinion is a collection of public opinions. Public opinion is the basis of social behavior, can accelerate or hinder the settlement of social problems. The society is an open complex system, the public opinion is the inherent characteristics of system, communication entertainment years shows the complex form, become a complex problem, a typical social system in the information age, producing each opinion located in an increasingly prominent role, a profound impact on international political, economic, cultural and other fields of science and technology. It is of great theoretical value and practical significance to study the nature of public opinion in the community, and to study how to control and guide public opinion.

The traditional mathematical model usually describes propagation behavior of a set of deterministic differential equations, a basic assumption is that the relationship between people in society as a regular network, in recent years, the study of complex system theory provides a new modeling method beyond reduction theory: set some rules for the system, make the system of spontaneous evolution in certain circumstances, then investigate some properties emerge during system evolution. The micro discrete dynamic model of individual local spatial interaction instead of the whole macro based on this model, a very effective means to become the overall behavior of complex system research, has made a lot of achievements in computer virus spread, the spread of infectious diseases, in crisis communication.

Evolution of public opinion with the well-known public opinion dynamics model:

S model: describe two individuals who want to have the same opinion to persuade their neighbors to adopt the same opinion;

D model, randomly select one and their collaborators, to adjust their own public opinion tends to a common opinion;

$\mathrm{KH}$ model, the public opinion is divided into a number of small groups, and then each small group of public opinion as the next moment of public opinion to further study.

Public opinion dynamics model of the evolution of public opinion mechanism can be divided into two categories: (1) in which individuals familiar with each other in the network, each individual belief based on their neighbors opinion; (2) many small group network construction with the same faith to divide, and ask each other familiar or not, in order to reflect the reality of Holme and Newman proposed a new model in 2006, its evolution mechanism is the comprehensive evolution mechanism. 
In recent years, it has become a new trend to combine dynamical behavior with complex networks. There are people on the regular network, small world network on the evolution of public opinion, but also have BA use a scale-free network to study it, such as scale-free network irreversible dissemination of public opinion, public opinion propagation without random dynamic scaling on the network, they use a mechanism similar to the $\mathrm{S}$ model. Without considering the extension of network connection complement structure and public opinion.

The last person to BA scale-free as the background, the public opinion of the dynamic evolution of the evolution mechanism, which is in the process of evolution, changing network structure, and the evolution of the order parameter or random parameters describe the evolution process of the evolution of public opinion. And found the following characteristics:

the evolution of public opinion affects the network structure, the network structure is no longer the initial scale-free network;

the close relationship between the neighbors of the network is increasing, which is beneficial to the convergence effect of public opinion;

the overall trend of the community is reduced, and the average number of small societies is increasing;

with the passage of time, the evolution of public opinion has a strong convergence effect.

This paper will use the modeling of complex systems thinking to establish a system simulation of free Internet dissemination of public opinion, in order to accord with the reality of public opinion originated from several degree maximum point; this paper will use the dynamic evolution mechanism of public opinion to construct a consistent evolution model to simulate the competition of Internet public dissemination; to construct a communication model the ideal of Internet public opinion.

\section{A Trend Model of Internet Public Opinion}

\section{Assumptions about Internet public opinion}

Internet public opinion communication can be divided into two parts: free communication and competitive communication. Free communication is spread from the source of public opinion by certain rules, until the spread of public opinion integrity of the system. The competition is spread at the end of two after the free dissemination of public opinion but hold different but connected nodes in accordance with certain rules of the mutual assimilation system until no longer hold different opinion and connected to the node. Public opinion bundle of public opinion is divided into 1 and -1 , $\mathrm{G}(\mathrm{H})$ said the node $\mathrm{h}$ with public opinion $(\mathrm{g}(\mathrm{H})=1$ or -1$)$, the other total node to use $\mathrm{N}$ to express, this article $\mathrm{N}=200$. $\mathrm{B}(\mathrm{H})$ to express the influence coefficient of node $\mathrm{H}$.

\section{Construction of BA network}

The initial set of $\mathrm{M}(0)$ isolated nodes, and then continue to introduce new nodes are connected to the $\mathrm{m}$ already exists on the node, where the $\mathrm{m}<=\mathrm{m}(0)$. For the sake of determination, this paper takes $\mathrm{m}=\mathrm{m}(0)=4$.

The probability $\mathrm{H}(\mathrm{I})$, which is connected to an existing node $\mathrm{i}$, adopts a preferential connection mechanism to satisfy the following relations:

The node $\mathrm{I}$ is $\mathrm{K}(\mathrm{I})$, the degree of node $\mathrm{j}$ is $\mathrm{K}(\mathrm{J})$. If greater than or equal to the two points, or not connected.

\section{Free propagation model}

There are a large number of uncertain factors affecting the spread of public opinion. Individual's ideology, social background and even psychological factors, interaction between media and exchange between individuals, between individuals a lot of opinions and attitude change, reflects the complexity and uncertainty characteristics.

This study is only a preliminary communication model, taking into account the above effects of uncertain factors and characteristics of public opinion in the social reality of the US for free opposite opinion propagation model to make the following simplifying assumptions of public opinion: 
Give each node i a personal coefficient b (I) (where B (I) is greater than 0 and less than or equal to 1). 1 because of social opinion in general and the relationship more influential people began to spread, so we choose the system more moderate as individual nodes and the node source coefficient of public opinion with relatively large. In this paper, the 6 largest nodes are chosen as the source of public opinion, and the other one is a kind of public opinion and the other is the other one of the 3 nodes of the public opinion of the other 3 .

Suppose each individual s (I) $(\mathrm{i}=1,2), \ldots \mathrm{N})$, there are three kinds of attitude, respectively by the number 1,0 and 1 for the -1 to support an opinion, -1 to support the opposition of public opinion, 0 representative neutral.

At the beginning, the nodes outside the source of public opinion, so that their public opinion for the immediate $(\mathrm{s}=0)$.In a $\mathrm{s}$ is not equal to the node I 0 select a contact with it. And the public opinion for the neutral node, if the individual coefficient of B (I) B (J) minus the individual coefficients greater than or equal to the public opinion dissemination coefficient $\mathrm{m}(\mathrm{m}$ as a public opinion to determine whether can spread from one node to another factor. A node in $\mathrm{M}$ equal to $0.14) \mathrm{J}$ node for the I to change the public opinion public opinion. If $\mathrm{B}(\mathrm{I})$ is less than $\mathrm{B}(\mathrm{J})$, we do not make any changes.

In the third step after the completion of a public opinion if there are 0 nodes, the node and its adjacent point values, if the sum of the value is greater than 0 , so this section point value is 1 ; if the added value is less than 0 , makes a point of view the node value is 0 ; if after adding value is equal to 0 when I is odd I public opinion value is 1 , when I is even I public opinion value is 0 .

\section{Competition and communication of public opinion}

Public opinion is in the free dissemination of public opinion dissemination competition after the end of the two hold a different opinion but are connected to the nodes in accordance with certain rules of the mutual assimilation system until no longer hold different opinion and connected to the node communication mode.

The evolution model of public opinion based on scale-free network structure is obtained:

The evolution of public opinion affects the network structure, the network structure is not the initial scale-free network;

The close contact between the neighbors of the network increases, which is conducive to the convergence effect of public opinion;

Highlight the general trend of the community to reduce the average number of small communities in the increase;

With the passage of time, the evolution of public opinion has a strong convergence effect.

The performance of the public opinion propagation competition all above characteristics of scale-free network structure change model based on the evolution of public opinion, so we can use the competition spread scale-free network structure change public opinion evolution model based on the simulation of opposite opinion.

According to the above, we make the following rules for the spread of public opinion:

In the initial stage of the evolution of public opinion, each node I is given an evolution coefficient $\mathrm{C}(\mathrm{I})$. In this paper, $\mathrm{C}$ (I) is equal to the B (I). Then give an evolutionary parameter a, a value of 0 to determine a certain number between the real, this paper makes a equal to 0.1 . In the free dissemination of public opinion in the opposition we have given each node in the network of public opinion to give $\mathrm{h}$ random number $\mathrm{G}(\mathrm{H})$. The evolution of later, select a i node in every step we are arbitrary $\mathrm{J}$, another node and random choice and he connected, if the two nodes and the serial number is the same, don't do anything, if two nodes of the public opinion value is different, we selected the two node evolution coefficient (I) and C C (J) and differential evolution parameter we have set a good a comparison will appear two possible evolution:

if the $\mathrm{C}(\mathrm{I})$ and $\mathrm{C}(\mathrm{J})$ is less than the evolution parameter will be between $\mathrm{a}, \mathrm{I}$ and $\mathrm{j}$ of the connection node node removed, and the remaining nodes (i.e. not including I, J node) randomly selects a node and the I node is connected to this node random selection requirements this node must have the same opinion and node ig (I) and the previously not connected. 
if the difference between $\mathrm{C}(\mathrm{I})$ and $\mathrm{C}(\mathrm{J})$ is greater than the evolution parameter a, the node i's opinion value is given to the node $\mathrm{j}$.

combined with the reality of society, we make it a rule that opposition to the evolution of public opinion behind, if the number of nodes holding a summing point public opinion view value is less than the public opinion of the source of two times. Then we think that the public opinion has no vitality, then we will change the value of the public opinion of these nodes and its opposition to public opinion values. That is to say, the public opinion has been defeated and eliminated in the competition of public opinion.

The scale-free network structure change public opinion evolution model of competition model of the opposite opinion is based on: 1) closely contact individual neighbors of the network increases again, which helps to increase the convergence effect of public opinion; 2) opposite opinion evolution to finally have a public opinion become stronger, and the other one a public reception weakened, and even death.

\section{References}

[1] Tang, J., et al., ArnetMiner: Extraction and Mining of Academic Social Networks. 2008.

[2] ResearchGate, wikipedia , http://zh.wikipedia.org/wiki/Researchgate, 2012.

[3] Zhang Yan-Chao etc., The research of information dissemination model on online social network. Acta Phys. Sin. Vol.60,No.5(2011): Page66-72.

[4] Newman, M.E.J., The structure and function of complex networks. 2003.

[5] LIU Chang-yu,etc.,Public Opinion Propagation Model Based on Small World Networks[J],Journal of System Simulation,2006-12. 